\title{
Structural properties of weak cotype 2 spaces
}

\author{
Piotr Mankiewicz Nicole Tomczak-Jaegermann
}

\begin{abstract}
Several characterizations of weak cotype 2 and weak Hilbert spaces are given in terms of basis constants and other structural invariants of Banach spaces. For finite-dimensional spaces, characterizations depending on subspaces of fixed proportional dimension are proved.
\end{abstract}

\section{Introduction}

Results of this paper concern weak cotype 2 spaces and weak Hilbert spaces. Both classes are important in the local theory of Banach spaces, by virtue of their connection to the existence of large Euclidean subspaces. Recall that spaces of weak cotype 2 have been introduced by V. D. Milman and G. Pisier in M-P as spaces such that every finite dimensional subspace contains a further subspace of a fixed proportional dimension which is well Euclidean. This class has numerous other characterizations, either by geometric invariants combined with linear structure, or by inequalities between various ideal norms of related operators and their $s$-numbers (see e.g., [P.3] and references therein).

On the other hand, recent results of the authors ("M-T.1], M-T.3) relate certain structural invariants of proportional dimensional quotients of a finite-dimensional space to volumetric invariants of the space; thus, via the well developed theory, to the existence of Euclidean subspaces or quotients. For example, finite-dimensional results in M-T.3 imply, although it is not explicitely stated in the paper, that if a Banach space has the property that

Supported in part by KBN (Poland), NSERC (Canada) and CRF (Canada, University of Alberta). 
its all subspaces have a basis with a uniform upper bound for the basis constant, then the space is of weak cotype 2. This property is obviously much too strong to characterize Banach spaces of weak cotype 2; for instance, spaces $L_{p}$ (with $p \neq 2$ ) contain subspaces without approximation property, hence without basis. A natural question then arises whether a weaker condition involving structural invariants of the same type can in fact characterize spaces of weak cotype 2 . To put it more precisely, whether it is possible to replace in the original weak cotype 2 definition, the property of being "well Euclidean" by (much weaker) properties of having some structural invariants "well bounded".

One of the results of the present paper (Theorem 4.1 and the remark after Theorem 4.2) shows that this is indeed possible. There exists $\delta_{0}>0$ such that a Banach space $X$ is of weak cotype 2 if and only if every finite dimensional subspace $E$ contains a a subspace $E_{0} \subset E$ with $\operatorname{dim} E_{0} \geq \delta_{0} \operatorname{dim} E$ such that certain structural invariants (of $E_{0}$ ) have a uniform upper bound. The invariants considered here are the basis constants or the complexification constants (for Banach spaces over reals only) or the symmetry constants or in fact some other related parameters.

In the theory of proportional-dimensional subspaces of finite-dimensional spaces is is sometimes of interest to deduce properties of a fixed $n$-dimensional space $X$ from an information on its all $\alpha n$-dimensional subspaces, with a fixed proportion $0<\alpha<1$. A fundamental example is well known and follows from the theory of type and cotype: if all $\alpha n$-dimensional subspaces of $X$ are $C$-Euclidean than $X$ itself is $f(C)$-Euclidean (cf.e.g., [T]). A recent more difficult example can be found in [B] and [M-T.1]. In Section 5 we study $n$-dimensional spaces $X$ such that the basis constant of an arbitrary $\alpha n$-dimensional subspace $E \subset X$ satisfies $\mathrm{bc}(E) \leq C$. This leads to proportional dimensional versions of a result mentioned above which follows from M-T.3]. In particular we show that the bound $\mathrm{bc}(E), \mathrm{bc}(F) \leq C$ for all $\alpha n$-dimensional subspaces $E$ and all $\alpha n$-dimensional quotients $F$ of $X$ implies that $X$ is a weak Hilbert space, with the constant bounded above by a function of $C$.

The arguments in the paper are based on two related ideas. The first one is a technique developed in M-T.1 and [M-T.3 of finding rather strange finite dimensional subspaces in spaces which fail to have weak cotype 2 . In the dual setting, which is more convenient to use, it can be described as follows. First, for a finite dimensional Banach space $E$, using deep facts 
from the local theory of Banach spaces, we find a quotient $F$ which can be placed in a special position in $\mathbb{R}^{N}$; and next we use a random (probabilistic) argument in order to prove that majority of quotients of $F$ enjoys relative lack of well bounded operators. The second idea comes from M-T.2, where the authors have proved that a random proportional-dimensional quotient $F$ of $l_{1}^{n}$ cannot be embedded into a Banach space $F_{1}$ with a nice Schauder basis and $\operatorname{dim} F_{1} \leq(1+\delta) \operatorname{dim} E$ for some small fixed $\delta$.

The paper is organized as follows. In Section 2 we collect a background material related to geometry and local theory of Banach spaces. In Section 3 we discuss spaces with few well bounded operators and related volumetric lower estimates. The main results of the paper are proved in Sections 4 and 5. Section 6 contains a proof of a random result which generalizes the result from [M-T.2 to arbitrary finite-dimensional Banach spaces.

We shall consider only Banach spaces over reals. The complex case can be delt with in an analogous manner. Our notation will follow $\mathbb{P}$.3 $]$ and [T]. We also refer the reader to $\mathrm{P} .3$ for more details on Banach spaces of weak cotype 2 .

\section{Preliminaries}

We will use the following geometric definition of the weak cotype 2 spaces. A Banach space $X$ is of weak cotype 2 whenever there exist $0<\delta_{0}<1$ and $D_{0} \geq 1$ such that every finite-dimensional subspace $E$ of $X$ contains a subspace $\tilde{E} \subset E$ with $\operatorname{dim} \tilde{E}=k=\geq \delta_{0} \operatorname{dim} E$ and with the Banach-Mazur distance satisfying $\mathrm{d}\left(\tilde{E}, l_{2}^{k}\right) \leq D_{0}$. This definition is equivalent to the one most commonly used at present; in fact, it is shown in [P.3], Theorem 10.2, that the weak cotype 2 constant of $X$ satisfies

$$
w C_{2}(X) \leq C \delta_{0}^{-1} D_{0},
$$

where $C$ is a universal constant.

Recall that a Banach space $X$ is of weak type 2 whenever $X^{*}$ is of weak cotype 2 and $X$ is $K$-convex. In particular, the weak type 2 constant satisfies

$$
w T_{2}(X) \leq K(X) w C_{2}\left(X^{*}\right) .
$$

A Banach space $X$ is a weak Hilbert space if $X$ is of weak type 2 and of weak cotype 2. We have no use of the technical definition of the weak Hilbert 
constant, let us just recall that this constant is controlled from above by $w T_{2}(X)$ and $w C_{2}(X)$. Finally, the following inequality is an easy consequence of the result of Pisier from [P.2], Theorem 2 and Corollary 9, which in turn are related to Pisier's deep $K$-convexity theorem. If $X$ is a weak Hilbert, then the $K$-convexity constant of $X$ satisfies, for any $0<\theta \leq 1$,

$$
K(X) \leq C(\theta)\left(w T_{2}(X) w C_{2}(X)\right)^{\theta} .
$$

Let $(E,\|\cdot\|)$ be an $n$-dimensional Banach space. For the Banach-Mazur distance $\mathrm{d}\left(E, L_{2}^{n}\right)$ from $E$ to the Euclidean space $l_{2}^{n}$ we will use a shorter notation of $\mathrm{d}_{E}$. Fix a Euclidean norm $\|\cdot\|_{2}$ on $E$ and identify $E$ with $\mathbb{R}^{n}$ in such a way that $\|\cdot\|_{2}$ becomes the natural $l_{2}$-norm on $\mathbb{R}^{n}$. Let us recall that the volume ratio of $E, \operatorname{vr}(E)$, is defined by

$$
\operatorname{vr}(E)=\left(\operatorname{vol} B_{E} / \operatorname{vol} \mathcal{E}_{\max }\right)^{1 / n}
$$

where $\mathcal{E}_{\max } \subset B_{E}$ is the ellipsoid of maximal volume contained in $B_{E}$.

More generally, for any ellipsoid $\mathcal{E} \subset B_{E}$, let $|\cdot|_{2}$ be the associated Euclidean norm, and let $\rho=\left(\operatorname{vol} B_{E} / \operatorname{vol} \mathcal{E}\right)^{1 / n}$. Szarek's volume ratio result (cf.e.g., [P.3 Theorem 6.1) says that for any $1 \leq k<n$, there exists a subspace $H \subset E$ with $\operatorname{dim} H=k$ such that

$$
c \rho^{-n /(n-k)}|x|_{2} \leq\|x\| \leq|x|_{2} \text { for } x \in H,
$$

where $c>0$ is a universal constant. It should be mentioned that some other volumetric invariants allow estimates with much better asymptotic dependence on $\lambda=k / n$, as $\lambda \rightarrow 1$ (cf.e.g., $\mathbb{P} .3 \|$ ). However application of these more delicate methods would complicate proofs without making essential improvements to final inequalities.

For $k \leq n$ set

$$
V_{k}(E)=V_{k}\left(B_{E}\right)=\sup \left\{\left(\operatorname{vol} P_{F}\left(B_{E}\right) / \operatorname{vol} P_{F}\left(B_{2}^{n}\right)\right)^{1 / k} \mid F \subset E, \operatorname{dim} F=k\right\} .
$$

This invariant is related to the notion of volume numbers of operators $(c f .[\mathbb{P} .3]$ Chapter 9). In particular, $V_{k}(E) \leq V_{l}(E)$ for $1 \leq l \leq k \leq n$.

The relevance of this invariant to the problem of finding Euclidean quotients of $E$ is described by the following standard lemma. Its proof is based on Santalò inequality and the volume ratio method (2.4) used in the dual space $E^{*}$. We leave further details to the reader. 
Lemma 2.1 Let $0<\beta<1$. Let $E=\left(\mathbb{R}^{n},\|\cdot\|\right)$ be a Banach space such that $B_{E} \subset B_{2}^{n}$ and let $a>0$ satisfies $V_{\beta n}(E) \geq a$. For every $\sigma>0$ there is a quotient $G$ of $E$ such that $\operatorname{dim} G \geq \beta \sigma(1+\sigma)^{-1} n$ and $\mathrm{d}_{G} \leq C a^{-(1+\sigma)}$, where $C \geq 1$ is a universal constant.

The following fact is an easy consequence of [P.3], Lemma 8.8. Let $\mathcal{E}$ be an ellipsoid on $E$ and $F$ be a quotient of $E$ with $\operatorname{dim} F=\lambda n$ and with the quotient map $Q: E \rightarrow F$. Then

$$
\left(\operatorname{vol} Q\left(B_{E}\right) / \operatorname{vol} Q(\mathcal{E})\right)^{1 / \lambda n} \leq a(\lambda)\left(\operatorname{vol} B_{E} / \operatorname{vol} \mathcal{E}\right)^{1 / n},
$$

where $a(\lambda) \geq 1$ depends on $\lambda$ only. In particular, $\operatorname{vr}(F) \leq a(\lambda) \operatorname{vr}(E)$.

The next lemma shows that given a finite-dimensional Banach space one can dramatically improve geometric properties of its unit ball by passing to quotients of proportional dimensions. The argument is based on several deep results in the local theory of Banach spaces ([Mi], [B-S], cf. also [B.3] ).. The lemma implicitly uses the ellipsoid of minimal volume containing the unit ball $B_{E}$ of a given $n$-dimensional space $E$; in fact it is concerned with the following property of a Euclidean norm $|\cdot|_{2}$ on $E$ : there exists $c>0$ such that every rank $k$ orthogonal projection $P$ in $\left(E,|\cdot|_{2}\right)$ satisfies

$$
\left\|P: E \rightarrow\left(E,|\cdot|_{2}\right)\right\| \geq c(k / n)^{1 / 2} .
$$

The norm $\||\|| \mid$ associated to the minimal volume ellipsoid satisfies (2.6) with $c=1$ (cf.e.g., [T] Proposition 3.2.10).

Note that if $E$ is a finite-dimensinal Banach space and $|\cdot|_{2}$ is an Euclidean norm on $E$ then each qoutient map $Q: E \rightarrow F$ induces on $F$ in a natural way an Euclidean norm $|\cdot|_{2, F}$. Namely, we set $B_{2, F}=Q\left(B_{2, E}\right)$, where $B_{2, F}$ and $B_{2, F}$ stand for Euclidean balls in $F$ and $E$ respectively.

Lemma 2.2 For every $0<\lambda<1$ there is $\rho=\rho(\lambda) \geq 1$ and for every $c>0$ there is $\tilde{\kappa}=\tilde{\kappa}(\lambda, c) \geq 1$, such that the following conditions hold for any $n$-dimensional Banach space $E$ :

(i) There exists a Euclidean norm $|\cdot|_{2}$ on $E$, with the unit ball $B_{2}$, such that

$$
\left(2^{1 / 2} \mathrm{~d}_{E}\right)^{-1} B_{2} \subset B_{E} \subset B_{2}
$$

and it satisfies (2.9) with $c=2^{-1 / 2}$; 
(ii) there exists a $\lambda$-dimensional quotient $F$ of $E$ satisfying $\operatorname{vr}(F) \leq \rho$;

(iii) if $|\cdot|_{2}$ is a Euclidean norm on E satisfying (2.0) for some $c>0$, then there exists a $\lambda$-dimensional quotient $\tilde{F}$ of $E$ and an orthonormal basis $\left\{x_{i}\right\}$ in $\left(\tilde{F},|\cdot|_{2}\right)$ such that

$$
\max _{i}\left\|x_{i}\right\|_{\tilde{F}} \leq \tilde{\kappa}
$$

Proof The proof of $(i i)$ and $(i i i)$ was already given in [M-T.1], Proposition 3.5. As for $(i)$, the Euclidean norm described in this condition combines properties of the norm ||$\cdot|| \mid$ associated with the ellipsoid of minimal volume and a norm which determines the Euclidean distance $\mathrm{d}_{E}$. Indeed, let \|\|$\cdot\|\|^{\prime}$ be a norm satisfying

$$
\|x\|^{\prime} \leq\|x\| \leq \mathrm{d}_{E}\|x\|^{\prime} \quad \text { for } \quad x \in E .
$$

Then for $x \in E$ set

$$
|x|_{2}=2^{-1 / 2}\left(\left\||| x||^{2}+\right\|\|x\|^{\prime 2}\right)^{1 / 2} .
$$

Clearly, $|x|_{2} \leq\|x\| \leq 2^{1 / 2} \mathrm{~d}_{E}|x|_{2}$, for $x \in E$, hence (2.7) holds. To prove that $|\cdot|_{2}$ satisfies (2.6), observe that for every $x \in E$ and every rank $k$ orthogonal projection $P$ in $\left(E,|\cdot|_{2}\right)$ one has

$$
\begin{aligned}
|P x|_{2} & =2^{-1 / 2}\left(\|P x\|^{2}+\|P x\|^{\prime 2}\right)^{1 / 2} \\
& \geq 2^{-1 / 2}\|P x\| \geq 2^{-1 / 2}\left\|P_{1} P x\right\|=2^{-1 / 2}\left\|P_{1} x\right\|,
\end{aligned}
$$

where $P_{1}$ is the orthogonal projection in $(E,\|\| \cdot \| \mid)$ with $\operatorname{ker} P_{1}=\operatorname{ker} P$ and next apply (2.6) in the space $(E,|\|\cdot \mid\|)$.

If $F$ is a finite-dimensional space and $B_{2}$ is a Euclidean ball on $F$, then, for a fixed orthonormal basis $\left\{x_{i}\right\}$ in $F$, by $B_{1}$ we shall denote abs conv $\left\{x_{i}\right\}$.

Corollary 2.3 For every $0<\lambda<1$ there is $\rho=\rho(\lambda) \geq 1$ and $\kappa=\kappa(\lambda) \leq 1$, such that an arbitrary $n$-dimensional Banach space $E$ satisfies:

(i) there exist a $\lambda$-dimensional quotient $F$ of $E$ and a Euclidean ball $B_{2}$ on $F$ such that $\operatorname{vr}(F) \leq \rho$ and

$$
\kappa B_{1} \subset B_{F} \quad \text { and } \quad\left(2^{1 / 2} \mathrm{~d}_{E}\right)^{-1} B_{2} \subset B_{F} \subset B_{2} .
$$


(ii) there exist a $\lambda n / 2$-dimensional quotient $F$ of $E$ and a Euclidean ball $B_{2}$ on $F$ such that for some $\left(2^{1 / 2} \mathrm{~d}_{E}\right)^{-1} \leq a \leq 1$ we have

$$
\kappa B_{1} \subset B_{F}, \quad a B_{2} \subset B_{F} \subset B_{2}, \quad\left(\operatorname{vol} B_{F} / \operatorname{vol}\left(a B_{2}\right)\right)^{1 / \lambda n} \leq \rho .
$$

Proof Condition (i) follows directly from Lemma 2.2 by chosing a Euclidean norm on $E$ satisfying (2.7) and then passing twice to quotient spaces and using (2.5). Notice that a Euclidean ball $B_{2}$ on $E$ determines the natural Euclidean ball on every quotient $F$ on $E$, and if $B_{2}$ satisfies (2.6) and $\operatorname{dim} F$ is proportional to $\operatorname{dim} E$ then the ball on $F$ satisfies (2.6) as well, with the constant depending on the proportion.

To get (ii), fix $0<\lambda^{\prime}<1$ to be determined later. First pass to a $\lambda^{\prime} n$ dimensional quotient $F^{\prime}$ of $E$ satisfying Lemma 2.2 (i) and (ii). Let $\mathcal{E}$ be the maximal volume ellipsoid on $F^{\prime}$; there exists a quotient $F^{\prime \prime}$ of $F^{\prime}$ with $\operatorname{dim} F^{\prime \prime}=\operatorname{dim} F^{\prime} / 2$ and the quotient map $Q: F^{\prime} \rightarrow F^{\prime \prime}$ such that on $F^{\prime \prime}$ we have $Q(\mathcal{E})=a Q\left(B_{2}\right)$, for some $a$. Clearly, $a \leq 1$ and $(i)$ implies that $a \geq\left(2^{1 / 2} \mathrm{~d}_{E}\right)^{-1}$. Passing to a quotient $F$ of $F^{\prime \prime}$ with $\operatorname{dim} F=\lambda^{\prime} \operatorname{dim} F^{\prime \prime}$, and using Lemma 2.2 (iii), we get all required inclusions; the bound for the ratio of volumes follows from (2.5). Given $1 / 2<\lambda<1$ choose $\lambda^{\prime}$ such that $\operatorname{dim} F=\lambda n$, then all constants involved will depend on $\lambda$.

Volumetric techniques for finding Euclidean sections provide Euclidean subspaces of small proportional dimensions. The next lemma describes a method from [M-P] of constructing Euclidean subspaces of large proportional dimensions in spaces saturated with small Euclidean ones. The proof of the statement below can be found in M-T.1] Theorem 4.2.

Lemma 2.4 Let $0<\delta<\xi<1$. Let $Z$ be an $N$-dimensional space such that every $\xi N$-dimensional subspace $Z_{1}$ of $Z$ contains a subspace $H$ with $\operatorname{dim} H \geq(\xi-\delta) N$ such that $\mathrm{d}_{H} \leq D$, for some $D \geq 1$. Then for every $0<\eta<1-\delta$ there exists a subspace $\tilde{H}$ of $Z$ with $\operatorname{dim} \tilde{H} \geq(1-\delta-\eta) N$ such that $\mathrm{d}_{\tilde{H}} \leq c D$, where $c=c(\xi, \delta, \eta)$.

Let $E$ be an $n$-dimensional Banach space and let $\|\cdot\|_{2}$ be a Euclidean norm on $E$. Recall that an operator $T: E \rightarrow E$ is said to be $(k, \beta)$-mixing for $k, \beta \geq 0$, if and only if there is a subspace $F \subset E$ with $\operatorname{dim} F \geq k$ such that $\left|P_{F^{\perp}} T x\right|_{2} \geq \beta|x|_{2}$ for every $x \in F$, where $P_{F^{\perp}}$ denotes the the orthogonal 
projection onto $F^{\perp}$. If this is the case then we write $T \in \operatorname{Mix}_{n}(k, \beta)$. The fact whether a fixed operator $T$ is in $\operatorname{Mix}_{n}(k, \beta)$ may depend on the choice of the Euclidean structure on $E$. Clearly, if $k \geq \ell$, then $\operatorname{Mix}_{n}(k, \beta) \subset \operatorname{Mix}_{n}(\ell, \beta)$. The following proposition is a folklore one (cf.e.g., Sz.2 Lemma 3.4A, Ma.2]).

Proposition 2.5 Let $E$ be an n-dimensional Banach space. For an arbitrary Euclidean norm $|\cdot|_{2}$ on $E$ one has

(i) for every projection $P$ of rank $k \leq n / 2$, we have $2 P \in \operatorname{Mix}_{n}(k, 1)$;

(ii) if the basis constant $\mathrm{bc}(E) \leq M$ then for every $k \in \mathbb{I}$ there is an operator $T: E \rightarrow E$ with $\|T\| \leq 2 M$ and $T \in \operatorname{Mix}_{n}(k, 1)$;

(iii) let $T: E \rightarrow E, k \in I N$ and $\beta \geq 0$. Then $T \in \operatorname{Mix}_{n}(k, \beta)$ if and only if $T^{*} \in \operatorname{Mix}_{n}(k, \beta)$ (with respect to the dual Euclidean norm $\left.|\cdot|_{2}^{*}\right)$.

\section{Volumetric estimates}

Technical result which this paper is based upon yields the existence, for a given finite-dimensional Banach space, of a quotient space, say $F$, of proportional dimension which admits relatively few well bounded operators. Moreover the same property is satisfied in any further quotient $F_{0}$ of $F$ and in any space $\tilde{F}$ which admits $F$ as its quotient, provided that the dimension of the new spaces is close enough to the dimension of $F$.

In this section we shall work with an $N$-dimensional Banach space $E=$ $\left(\mathbb{R}^{N},\|\cdot\|\right)$, on which we always consider the (natural) Euclidean norm $\|\cdot\|_{2}$ and the associated Euclidean ball $B_{2}^{N}$. If $F$ is a quotient of $E$, with the quotient map $Q: E \rightarrow F$, then the natural Euclidean norm on $F$ has the unit ball $Q\left(B_{2}^{N}\right)$. Unless otherwise stated, these natural Euclidean norms are used for all geometric invariants.

First we discuss quotients which admit lower estimates for norms of mixing operators. The main analytic estimate is stated in the following theorem; the proof involves a random construction and it is postponed until Section 6 .

Theorem 3.1 For an arbitrary $0<\delta<1,0<\eta<3 / 8$ and $0<\varepsilon<2^{-5} \eta$, set $\gamma=2^{-5} \delta \varepsilon \eta$, and for $n \in \mathbb{I N}$ set $N=(1+\varepsilon) n$. Let $E=\left(\mathbb{R}^{N},\|\cdot\|\right)$ be an 
$N$-dimensional Banach space such that $B_{E} \subset B_{2}^{N}$. Let $\rho \geq 1,0<\kappa \leq 1$ and $0<a \leq 1$ satisfy

$$
\operatorname{vr}(E) \leq \rho, \quad \kappa B_{1}^{N} \subset B_{E}, \quad a B_{2}^{N} \subset B_{E} .
$$

Then $E$ admits an $n$-dimensional quotient $F$ such that for every operator $T: F \rightarrow F$ with $T \in \operatorname{Mix}_{n}(\eta n, 1)$, and every quotient map $Q: F \rightarrow Q(F)$ with rank $Q \geq(1-\gamma) n$, one has

$$
\|Q T: F \rightarrow Q(F)\| \geq c V_{\eta n / 4}(E)^{-1} a^{\delta},
$$

where $c=c(\varepsilon, \eta, \kappa, \rho)>0$.

Remark Theorem 3.1 remains valid also for $\delta=0$. In this case we have $\gamma=0$ (note that the constant $c$ does not depend on $\gamma$ ) and the theorem reduces to Theorem 2.2 from [M-T.3].

Formula 3.2 implies that the space $F$ as well as its further quotients $F_{0}$ admit relatively few well bounded operators. Indeed, one can formally deduce from it well bounded operators on these spaces are small perturbations of a multiple of the identity operator.

Proposition 3.2 With the same notation as used in Theorem 3.1 if $E=$ $\left(\mathbb{R}^{N},\|\cdot\|\right)$ is an $N$-dimensional Banach space such that $B_{E} \subset B_{2}^{N}$ and satisfying (3.1), then $E$ admits an $n$-dimensional quotient $F$ which satisfies the following two conditions:

(i) for every quotient $F_{0}$ of $F$ with $\operatorname{dim} F_{0}=k \geq(1-\gamma) n$ and every operator $T: F_{0} \rightarrow F_{0}$, with $T \in \operatorname{Mix}_{k}\left(\eta_{0} k, 1\right)$, where $\eta_{0}=\eta /(1-\gamma)$, we have

$$
\|T\| \geq c V_{\eta n / 4}(E)^{-1} a^{\delta},
$$

(ii) every Banach space $\tilde{F}$ with $\operatorname{dim} \tilde{F}=l \leq(1+\gamma) n$ such that $F$ is a quotient of $\tilde{F}$ admits a Euclidean norm such that every operator $\tilde{T}$ : $\tilde{F} \rightarrow \tilde{F}$, which is $(\tilde{\eta} l, 1)$-mixing with respect to this norm, where $\tilde{\eta}=$ $(\eta+2 \gamma)$, satisfies

$$
\|\tilde{T}\| \geq c V_{\eta n / 4}(E)^{-1} a^{\delta} .
$$

Here $c=c(\varepsilon, \eta, \kappa, \rho)>0$. 
Proof Let $F$ be the quotient of $E$ satisfying Theorem 3.1. In particular, $F$ admits the natural Euclidean norm inherited from $E$. Set

$$
K=\inf _{Q} \inf \left\{\|Q \tilde{T}: F \rightarrow Q(F)\| \mid \tilde{T}: F \rightarrow F, \tilde{T} \in \operatorname{Mix}_{n}(\eta n, 1)\right\},
$$

where the first infimum runs over all quotient maps $Q: F \rightarrow Q(F)$ with rank $Q \geq(1-\gamma) n$. To prove $(i)$, pick a quotient $F_{0}=F / G_{0}$, with $\operatorname{dim} F_{0}=$ $k \geq(1-\gamma) n$. Identify $F_{0}$ with the linear subspace $G_{0}^{\perp} \subset F$, under the norm whose unit ball is $Q_{G_{0}}\left(B_{F}\right)$; here $Q_{G_{0}}$ is the othogonal projection with $\operatorname{ker} Q_{G_{0}}=G_{0}$. Fix an arbitrary $\left(\eta^{\prime} k, 1\right)$-mixing operator $T: F_{0} \rightarrow F_{0}$. Pick any $\tilde{T}: F \rightarrow F$ such that $Q_{G_{0}} \tilde{T}=T Q_{G_{0}}$. Since for $x \in G_{0}^{\perp}$ we have $\tilde{T} x=T x+z$, for some $z \in G_{0}$, then $\tilde{T} \in \operatorname{Mix}_{n}\left(\eta^{\prime} k, 1\right)$. Since $\eta^{\prime} k \geq \eta n$, then $\tilde{T} \in \operatorname{Mix}_{n}(\eta n, 1)$. Thus, by the definition of $K$ we have

$$
\left\|T: F_{0} \rightarrow F_{0}\right\|=\left\|Q_{G_{0}} T Q_{G_{0}}: F \rightarrow F_{0}\right\|=\left\|Q_{G_{0}} \tilde{T}: F \rightarrow F_{0}\right\| \geq K,
$$

and $(i)$ by the estimate (3.2).

The proof of $(i i)$ is very similar. Fix an $l$-dimensional space $\tilde{F}$ with $l \leq(1+\gamma) n$ such that $q_{F}: \tilde{F} \rightarrow F$ is the quotient map. Consider an arbitrary Euclidean norm on $\tilde{F}$, with the unit ball $\tilde{B}_{2}$, such that $q_{F}\left(\tilde{B}_{2}\right)$ is the natural Euclidean ball on $F$. Let $\tilde{T}: \tilde{F} \rightarrow \tilde{F}$ be $(\tilde{\eta} l, 1)$-mixing. If $T: F \rightarrow F$ satisfies $T q_{F}=q_{F} \tilde{T}$ then clearly, $\|\tilde{T}\| \geq\left\|q_{F} \tilde{T}\right\|=\|T\|$. Moreover, since $\operatorname{dim} \operatorname{ker} q_{F} \leq \gamma n$, then $T \in \operatorname{Mix}_{n}(\tilde{\eta} l-2 \gamma n, 1) \subset \operatorname{Mix}_{n}(\eta n, 1)$. Thus $\|T\| \geq K$ and the lower estimate for $\|\tilde{T}\|$ follows.

It is well known that if for a space $F$ all mixing operators have large norms, then $F$ itself and other related spaces have several structural invariants, such as basis constant or symmetry constant or complexification constant, also bounded below (cf.e.g., M-T.3 Section 6). We give an example of an estimate of this type.

Corollary 3.3 For an arbitrary $0<\varepsilon<2^{-13}$ and $0<\delta<1$, set $\gamma=2^{-13} \varepsilon \delta$, and for $n \in \mathbb{N}$ set $m=(1+2 \varepsilon) n$. Let $E$ ba an $m$-dimensional Banach space and let $\mathrm{d}_{E}=\mathrm{d}\left(E, l_{2}^{m}\right)$. There exists an n-dimensional quotient $F$ of $E$ such that for any further quotient $F_{0}$ of $F$ with $\operatorname{dim} F_{0} \geq(1-\gamma) n$, and for any space $\tilde{F}$ with $\operatorname{dim} \tilde{F} \leq(1+\gamma) n$, for whose $F$ is a quotient, denoting by $F^{\prime}$ either $F_{0}$ or $\tilde{F}$, we have

$$
\mathrm{bc}\left(F^{\prime}\right) \geq c V_{2^{-10} n}(E)^{-1} \mathrm{~d}_{E}^{-\delta} .
$$


Moreover, for any Banach space $Z$ such that every $n$-dimensional subspace of $Z$ is D-Euclidean we have

$$
\mathrm{bc}\left(F^{\prime} \oplus_{2} Z\right) \geq c D^{-1} V_{2^{-10} n}(E)^{-1 / 2} \mathrm{~d}_{E}^{-\delta / 2} .
$$

Here $c=c(\varepsilon, \delta)>0$.

The choice of $\eta=2^{-8}$ made below when applying Proposition 3.2 was done for convenience of past references. With appropriate modifications the same argument would work for an arbitrary $0<\eta<3 / 8$.

Proof By Corollary $2.3(i)$, there exists a $(1+\varepsilon) n$-dimensional quotient $\tilde{E}$ of $E$ and a Euclidean ball on $\tilde{E}, \tilde{B}_{2} \supset B_{\tilde{E}}$, such that (3.1) holds, with some $\rho=\rho(\varepsilon), \kappa=\kappa(\varepsilon)$ and $a=\left(2^{1 / 2} \mathrm{~d}_{E}\right)^{-1}$.

Fix $\eta=2^{-8}$. Then operators on $F^{\prime}$ which are $\left(5 \cdot 2^{-9} \operatorname{dim} F^{\prime}, 1\right)$-mixing are also $\left(\eta^{\prime} \operatorname{dim} F^{\prime}, 1\right)$-mixing, where $\eta^{\prime}=\eta_{0}$ or $\eta^{\prime}=\tilde{\eta}$, depending on the choice of $F^{\prime}$ being $F_{0}$ or $\tilde{F}$. Therefore, by Proposition 3.2 , these operators have norms bounded below by $K=c V_{2^{-10} n}(E)^{-1} \mathrm{~d}_{E}^{-\delta}$. Thus the conclusion follows from Theorem 2.1 in [M-T.3].

Remark The quotient space $F$ itself satisfies (3.5) and (3.6) as well, with $\delta=0$. This was the content of Theorem 2.4 in M-T.3], and it followed from the construction in M-T.3, Theorem 2.2, which preceded the present construction.

It is of independent interest to study a relationship between various $s$ numbers of operators acting in spaces discussed in Theorem 3.1. The advantage of this approach lies in the fact that resulting estimates are valid for all operators, and not only for mixing ones.

Let us recall relevant definitions. Let $X$ and $Y$ be Banach spaces and let $T: X \rightarrow Y$ be a bounded operator. Let $k$ be a positive integer. The $k$ th Kolmogorov number $d_{k}(T)$ is defined by

$$
d_{k}(T)=\inf _{Z \subset Y} \sup _{x \in B_{X}} \inf _{y \in Z}\|T x-y\|,
$$

where the infimum on $Z$ runs over all subspaces $Z$ of $Y$ with $\operatorname{dim} Z<k$. The dual concept is that of Gelfand numbers which are defined by

$$
c_{k}(T)=\inf \left\{\left\|\left.T\right|_{Z}\right\| \mid Z \subset X, \operatorname{codim} Z<k\right\} .
$$

We have $c_{k}(T)=d_{k}\left(T^{*}\right)$ for arbitrary $X$ and $Y$ and $T$.

As a consequence of Theorem 3.1 we get. 
Proposition 3.4 For an arbitrary $0<\varepsilon<2^{-10}$ and $0<\delta<1$ set $\gamma=$ $2^{-10} \delta \varepsilon$, and for $n \in \mathbb{I}$ set $N=2(1+2 \varepsilon) n$. Let $E=\left(\mathbb{R}^{N},\|\cdot\|\right)$ be an $N$-dimensional Banach space. Then $E$ admits an $n$-dimensional quotient $F$ such that for every operator $T: F \rightarrow F$ we have

$$
d_{\gamma n}(T) \geq c V_{2^{-7} n}(E)^{-1} \mathrm{~d}_{E}^{-\delta} \inf _{\lambda \in \mathbb{R}} c_{n / 4}\left(T-\lambda \mathrm{Id}_{F}\right),
$$

where $c=c(\varepsilon)>0$.

Proof Let $F_{0}$ be a $(1+\varepsilon) n$-dimensional quotient of $E$ satifying condition (ii) of Corollary 2.3, for a Euclidean ball $B_{2}$ and some $0<\kappa=\kappa(\varepsilon) \leq 1$, $\rho=\rho(\varepsilon)$ and $0<a \leq 1$, Let $F$ be a quotient of $F_{0}$ satisfying (3.2) of Theorem 3.1, with $\eta=2^{-5}$. Let $Q: F_{0} \rightarrow F$ be the quotient map. Let be the Euclidean norm on $F$ corresponding to $Q\left(B_{2}\right)$; in particular, $\|x\| \leq a^{-1}\|x\|_{2}$ for $x \in F$. Then (2.5) implies that

$$
\left(\operatorname{vol} B_{F} / \operatorname{vol} Q\left(a B_{2}^{N}\right)\right)^{1 / n} \leq C(\varepsilon) \rho .
$$

By the volume ratio argument, pick a 15n/16-dimensional subspace $H$ of $F$ such that

$$
A a^{-1}\|x\|_{2} \leq\|x\| \leq a^{-1}\|x\|_{2} \quad \text { for } \quad x \in H,
$$

with $A=A(\varepsilon)=(C(\varepsilon) \rho)^{16}$.

Fix an arbitrary $T: F \rightarrow F$ satisfying

$$
\inf \left\{\|\left.(T-\lambda \text { Id })\right|_{G} \|_{2} \mid \lambda \in \mathbb{R}, G \subset F, \operatorname{dim} G=7 n / 8\right\}=1 .
$$

Pick $G_{0} \subset F$ with $\operatorname{dim} G_{0}=7 n / 8$ and $\lambda_{0}$ such that $\left\|\left.\left(T-\lambda_{0} \mathrm{Id}\right)\right|_{G_{0}}\right\|_{2} \leq 2$. Set $G_{1}=\left\{x \in H \cap G_{0} \mid T x \in H\right\}$. Then $\operatorname{dim} G_{1} \geq 3 n / 4$. By (3.7) one has

$$
\left\|\left(T-\lambda_{0} \mathrm{Id}\right) x\right\| \leq 2 A\|x\| \quad \text { for } \quad x \in G_{1} .
$$

Hence $\left\|\left.\left(T-\lambda_{0} \mathrm{Id}\right)\right|_{G_{1}}\right\| \leq 2 A$, which means

$$
\inf _{\lambda \in \mathbb{R}} c_{n / 4}\left(T-\lambda \mathrm{Id}_{F}\right) \leq 2 A .
$$

On the other hand, the normalization condition for $T$ and Lemma 2.1 in [Ma.3] imply that $8 T$ is $\left(2^{-5} n, 1\right)$-mixing. Denoting the right hand side of (3.2) by $K$, we get

$$
\inf \|Q T: F \rightarrow Q(F)\| \geq K / 8,
$$


with the infimum taken over all quotient maps $Q: F \rightarrow Q(F)$ with $\operatorname{rank} Q \geq$ $(1-\gamma) n$. By the definition of Kolmogorov numbers this means that $d_{\gamma n}(T) \geq$ $K / 8$. Combining this estimate with (3.8) we conclude the proof.

\section{Characterizations of infinite-dimensional spaces}

As mentioned in the introduction, if a Banach space $X$ has the property that its all subspaces have a basis with a uniform upper bound for the basis constant, then $X$ is of weak cotype 2 . In fact, if there exists $M<\infty$ such that $\mathrm{bc}(E) \leq M$ for every subspace $E$ of $X$, then $w C_{2}(X)$ admits an upper estimate by a function of $M$. Indeed, let $F$ be an arbitrary finite-dimensional quotient of $X^{*}$ and consider the Euclidean structure of $F$ determined by the ellipsoid of minimal volume containing the unit ball $B_{F}$. Then the dual version of Theorem 2.4 in $\mathbb{M}-\mathrm{T} .3$ implies that $V_{\beta n}(F) \geq c / M$, for some universal constants $\beta>0$ and $c>0$. Then the conclusion follows immediately from Lemma 2.1, by passing back to the space $X$.

A similar general line of argument is used to prove characterizations of weak cotype 2 spaces in terms of the basis constant; we also obtain related characterizations in terms of an existence of uniformly bounded projections and mixing operators.

Theorem 4.1 There exists a constant $\gamma_{0}>0$ such that a Banach space $X$ is of weak cotype 2 if and only if there exist a constant $M \geq 1$ such that every finite-dimensional subspace $E$ of $X$ contains a subspace $E_{0} \subset E$ with $k=\operatorname{dim} E_{0} \geq\left(1-\gamma_{0}\right) \operatorname{dim} E$ satisfying one of the following conditions:

(i) $\mathrm{bc}\left(E_{0}\right) \leq M$,

(ii) there exists a projection $Q: E_{0} \rightarrow E_{0}$ of rank $k / 8$ such that $\|Q\| \leq M$,

(iii) for every Euclidean norm on $E_{0}$ there exists an operator $T: E_{0} \rightarrow E_{0}$ which is $(k / 8,1)$-mixing with respect to this norm, such that $\|T\| \leq M$.

Moreover, if one of the conditions (i)-(iii) holds then $w C_{2}(X) \leq C M^{4}$, where $C$ is a universal constant. 
The counterpart of this result for superspaces is less satisfactory, as it gives implication in one direction only.

Theorem 4.2 There exists a constant $\gamma_{0}>0$ such that whenever $X$ is a Banach space for which there exist a constant $M \geq 1$ such that for every finite-dimensional subspace $E$ of $X$ there is a Banach space $\tilde{E} \supset E$ with $k=\operatorname{dim} \tilde{E} \leq\left(1+\gamma_{0}\right) \operatorname{dim} E$ satisfying one of the following conditions

(i) $\mathrm{bc}(\tilde{E}) \leq M$,

(ii) there exists a projection $Q: E_{0} \rightarrow E_{0}$ of rank $k / 8$ such that $\|Q\| \leq M$,

(iii) for every Euclidean norm on $\tilde{E}$ there exists an operator $T: \tilde{E} \rightarrow \tilde{E}$ which is $(k / 8,1)$-mixing with respect to this norm, such that $\|T\| \leq M$,

then $X$ is of weak cotype 2 and $w C_{2}(X) \leq C M^{4}$, where $C$ is a universal constant.

Remark Beside conditions $(i)$ and $(i i)$ of the above theorem there is a number of other invariants whose uniform boundedness implies condition (iii). They are symmetry constant ( $c f$. Ma.1, Ma.2 ), complexification constant (for real Banach spaces) or the Banach-Mazur distance from the space to its complex conjugate (for complex Banach spaces) ( $c f$. [5z.2]). All these and other invariants could be used for versions of all theorems of this section ( $c f$. also M-T.3, Section 6).

Remark Note that there is an essential difference betwen the Euclidean case in the definition of weak cotype 2 and the results above. Namely, we do not know whether analogous characterizations are valid for an arbitrary proportion $\delta \in(0,1)$ (not necessary $\left.\delta>1-\gamma_{0}\right)$. Recall that it is so in the Euclidean case: if for a Banach space $X$ there exists $\delta_{0}>0$ such that every finite dimensional subspace $E$ of $X$ contains a $C_{0}$-Euclidean subspace $E_{0} \subset E$ with $\operatorname{dim} E_{0} \geq \delta_{0} \operatorname{dim} E$, then an analogous condition holds for every $\delta \in(0,1)$, with the constant $C$ depending on $\delta$. It seems that the present difficulty is connected with a problem of Pełczyński in Pe.1 whether every finite dimensional Banach space $E$ can be embeded into a Banach space $F$ with $\operatorname{dim} F \leq 2 \operatorname{dim} E$ having a nice Schauder basis. For $\operatorname{dim} F$ close enough to $\operatorname{dim} E$, this question was answered in M-T.2 in the negative. 
Proof of Theorem 4.1 Clearly, the weak cotype 2 assumption implies property $(i)$, which implies (ii), which implies (iii). We shall prove that conversely, property (iii) implies that $X$ is of weak cotype 2 . Set $\gamma_{0}=2^{-20}$. Under our assumptions we have the following.

Claim For every finite-dimensional subspace $E \subset X$ there exists a subspace $H \subset E$ with $\operatorname{dim} H \geq 2^{-9} \operatorname{dim} E$ and $\mathrm{d}_{H} \leq c M^{2} \mathrm{~d}_{E}^{1 / 2}$, where $c$ is a universal constant.

Proof of the Claim Let $\varepsilon=2^{-9}, \eta=2^{-4}, \delta=2^{-2}$, and $\gamma=\gamma_{0}$. Fix an arbitrary finite-dimensional $E \subset X$ and let $n \in \mathbb{N}$ be such that $\operatorname{dim} E=$ $(1+2 \varepsilon) n$.

Set $Z=E^{*}$. Let $Z_{1}$ be a quotient of $Z$ with $\operatorname{dim} Z_{1}=(1+\varepsilon) n$ satisfying condition $(i)$ of Corollary 2.3. We also fix the Euclidean structure on $Z_{1}$ introduced in this condition. Let $Z_{2}$, with $\operatorname{dim} Z_{2}=n$, be a quotient of $Z_{1}$ constructed in Proposition 3.2. Now we use the dual form of (iii) valid for every finite-dimensional quotient of $X^{*}(c f$. Proposition $2.5(i v))$. It follows that $Z_{2}$ has a quotient $Z_{3}$ with $\operatorname{dim} Z_{3}=k \geq(1-\gamma) n$ which admits a $(k / 8,1)$-mixing operator $S_{0}$ with $\left\|S_{0}\right\| \leq M$. On the other hand, by the choice of $Z_{2}$, every $\left(\eta^{\prime} k, 1\right)$-mixing operator $T$ on $Z_{3}$ satisfies

$$
\|T\| \geq c V_{2^{-6} n}^{-1}\left(Z_{1}\right) \mathrm{d}_{Z}^{-\delta},
$$

where $\eta^{\prime}=\eta /(1-\gamma)$. Since $\eta^{\prime} \leq 1 / 8$, the same estimate holds for $S_{0}$. Thus $V_{2^{-6}{ }_{n}}\left(Z_{1}\right) \geq c \mathrm{~d}_{Z}^{-\delta} M^{-1}$. Applying Lemma 2.1 with $\beta=2^{-6}$ and $\sigma=1$, we obtain a quotient $Z_{4}$ of $Z_{3}$ with $\operatorname{dim} Z_{4} \geq 2^{-7} k \geq 2^{-9} \operatorname{dim} E$ and $\mathrm{d}_{Z_{4}} \leq$ $C \mathrm{~d}_{Z}^{2 \delta} M^{2}$. Since $\mathrm{d}_{Z}=\mathrm{d}_{E}$, the proof of the Claim is concluded by setting $H=Z_{4}^{*}$.

Passing to the proof of the theorem, fix an arbitrary finite-dimensional subspace $X_{0} \subset X$. Denote by $D$ the smallest number such that every subspace $X_{1}$ of $X_{0}$ contains a subspace $G \subset X_{1}$ with $\operatorname{dim} G \geq \operatorname{dim} X_{1} / 2$ and $\mathrm{d}_{G} \leq D$. Clearly, $D$ is finite.

We then know that for any $m$ and any subspace $X_{1}$ of $X_{0}$ with $\operatorname{dim} X_{1}=$ $m$ there is a subspace $H \subset X_{1}$ with $\operatorname{dim} H \geq 2^{-10} m$ such that $\mathrm{d}_{H} \leq c M^{2} D^{1 / 2}$. Indeed, first pick $E \subset X_{1}$ with $\operatorname{dim} E \geq m / 2$ such that $\mathrm{d}_{E} \leq D$, and then apply Claim to obtain $H$. Specifying $m=(1 / 2) \operatorname{dim} X_{0}$ we can use Lemma 2.4 with $\xi=1 / 2, \delta=\left(1-2^{-10}\right) \xi$ and $\eta=2^{-10} \xi$, to get a subspace $\tilde{H} \subset X_{0}$ satisfying $\operatorname{dim} \tilde{H} \geq(1 / 2) \operatorname{dim} X_{0}$ and $\mathrm{d}_{\tilde{H}} \leq c^{\prime} M^{2} D^{1 / 2}$, where $c^{\prime}$ is a 
universal constant. By the definition of $D$, this implies $D \leq c^{\prime} M^{2} D^{1 / 2}$, hence $D \leq c^{\prime \prime} M^{4}$. By (2.1), this completes the proof.

Theorem 4.2 has almost identical proof, with condition (ii) of Proposition 3.2 replacing $(i)$. We shall omit further details.

Remark Using Lemma 2.1 in a more delicate way and choosing $\delta$ sufficiently small one can get in the theorems above $w C_{2}(X) \leq C(\sigma) M^{1+\sigma}$, for every $\sigma>0$.

We now pass to characterizations of weak Hilbert spaces.

Theorem 4.3 There exists a constant $\gamma_{0}>0$ such that a Banach space $X$ is a weak Hilbert space if and only if there exists a constant $M \geq 1$ such that one of the following conditions is satisfied for every subspace $Y \subset X$ :

(i) every finite-dimensional subspace $E$ of $Y$ contains a subspace $E_{0}$ with $\operatorname{dim} E_{0} \geq\left(1-\gamma_{0}\right) \operatorname{dim} E$ which admits a projection $Q: E_{0} \rightarrow E_{0}$ with $\operatorname{rank} Q=\operatorname{dim} E_{0} / 8$ and $\|Q\| \leq M$, and

every finite-dimensional quotient $F$ of $Y$ admits a quotient $F_{0}$ with $\operatorname{dim} F_{0} \geq\left(1-\gamma_{0}\right) \operatorname{dim} F$ which admits a projection $R: F_{0} \rightarrow F_{0}$ with $\operatorname{rank} R=\operatorname{dim} F_{0} / 8$ and $\|Q\| \leq M$,

(ii) for every finite-dimensional subspace $E$ of $Y$ there is a Banach space $\tilde{E}$ containing $E$ with $\operatorname{dim} \tilde{E} \leq\left(1+\gamma_{0}\right) \operatorname{dim} E$ which admits a projection $\tilde{Q}: \tilde{E} \rightarrow \tilde{E}$ with $\operatorname{rank} \tilde{Q}=\operatorname{dim} \tilde{E} / 8$ and $\|\tilde{Q}\| \leq M$, and

for every finite-dimensional quotient $F$ of $Y$ there is a Banach space $\tilde{F}$ which has $F$ as a a quotient and $\operatorname{dim} \tilde{F} \leq\left(1+\gamma_{0}\right) \operatorname{dim} F$ which admits a projection $R: \tilde{F} \rightarrow \tilde{F}$ with $\operatorname{rank} R=\operatorname{dim} \tilde{F} / 8$ and $\|R\| \leq M$.

Proof Clearly, if $X$ is a weak Hilbert space then both $(i)$ and $(i i)$ are satisfied. Conversely, assuming that one of conditions $(i)$ or $(i i)$ is satisfied, by Theorem 1 in $\mathbb{M - T . 2}, X$ does not contain $l_{1}^{n}$ 's uniformly. Hence, by Pisier's result, $\mathbb{P} .1],(c f$. also [P.3], Theorem 11.3), $X$ is $K$-convex.

If $(i)$ holds, by Theorem 4.1 we infer that both $X$ and $X^{*}$ are of weak cotype 2 . Thus $X$ is a weak Hilbert space. In case of $(i i)$, we use Theorem 4.2 .

Remark If $X$ has the approximation property, the above theorem remains valid if we restrict ourselves to the case $Y=X$ only. 
Remark In Theorem 4.3, the assumption on the existence of uniformly bounded rank $k / 8$ projections can be replaced by the existence of uniformly bounded $(k / 8,1)$-mixing operators or by a uniform bound for basis constants.

\section{Subspaces and quotients of proportional di- mension}

The main result of this section is concerned with subspaces and quotients of a fixed proportional dimension.

Theorem 5.1 Let $0<\alpha<\left(1+2^{-8}\right)^{-1}$ and let $M \geq 1$. Let $G$ be an $n$ dimensional Banach space. If every an-dimensional subspace $E$ and every $\alpha$-dimensional quotient $F$ of $G$ have the basis constants $\mathrm{bc}(E) \leq M$ and $\mathrm{bc}(F) \leq M$, then $G$ is a weak Hilbert space and the weak type 2 and the weak cotype 2 constants satisfy the estimates

$$
w T_{2}(G) \leq C(\alpha) M^{140 / 3} \quad \text { and } \quad w C_{2}(G) \leq C(\alpha) M^{100 / 3} .
$$

The proof of the theorem requires several steps. To begin with we consider only one-sided assumptions on $G$, that is, the assumptions on its subspaces. In such a situation, the following proposition establishes a weak cotype 2 property, provided that the space admits a nice direct sum decomposition.

Proposition 5.2 Let $0<\alpha<1, M \geq 1$ and let $G$ be an n-dimensional Banach space. Assume that $G=Z \oplus_{2} G_{0}$, with $\operatorname{dim} Z \geq \alpha$. If every andimensional subspace $E$ of $G$ has the basis constant $\operatorname{bc}(E) \leq M$, then the weak cotype 2 constant of $G_{0}$ satisfies $w C_{2}\left(G_{0}\right) \leq C(\alpha)\left(M \inf _{H} \mathrm{~d}_{H}\right)^{4}$, where the infimum runs over all an-dimensional subspaces $H \subset Z$.

Proof Let $H \subset Z$ be an $\alpha$-dimensional subspace. We shall show that every subspace $G_{1}$ of $G_{0}$ contains a subspace $\tilde{E}$, with $\operatorname{dim} \tilde{E} \geq 2^{-12} \min \left(\operatorname{dim} G_{1}, \alpha n\right)$ such that $\mathrm{d}_{\tilde{E}} \leq C\left(M \mathrm{~d}_{H}\right)^{4}$. By (2.1), this will imply $w C_{2}\left(G_{0}\right) \leq C(\alpha)\left(M \mathrm{~d}_{H}\right)^{4}$, hence the conclusion will follow by passing to the infimum over $H$. Obviously it is enough to consider the case $\operatorname{dim} G_{1} \leq \alpha n$ only.

Fix an arbitrary subspace $G_{1}$ of $G_{0}$ with $k=\operatorname{dim} G_{1} \leq \alpha n$ and let $F=G_{1}^{*}$. By the remark following Corollary 3.3 there exists a quotient $F_{1}$ of $F$ with $k^{\prime}=\operatorname{dim} F_{1} \geq\left(1+2^{-12}\right)^{-1} k$ satisfying (3.6) with $\delta=0$. Fix an 
arbitrary $H_{1} \subset H$ with $\operatorname{dim} H_{1}=\alpha n-k^{\prime}$. We have $\mathrm{d}_{H_{1}^{*}}=\mathrm{d}_{H_{1}} \leq \mathrm{d}_{H}$ and $H_{1}^{*}$ is a quotient of $Z$. Thus

$$
\operatorname{bc}\left(F_{1} \oplus_{2} H_{1}^{*}\right) \geq c \mathrm{~d}_{H}^{-1} V_{2^{-10} k^{\prime}}(F)^{-1 / 2} .
$$

Observe that $\left(F_{1} \oplus_{2} H_{1}^{*}\right)^{*}$ is an $\alpha n$-dimensional subspace of $G$. Hence bc $\left(F_{1} \oplus_{2}\right.$ $\left.H_{1}^{*}\right)=\operatorname{bc}\left(\left(F_{1} \oplus_{2} H_{1}^{*}\right)^{*}\right) \leq M$. Combining the last two estimates we get

$$
V_{2^{-10} k^{\prime}}(F) \geq c^{\prime}\left(M \mathrm{~d}_{H}\right)^{-2} \text {. }
$$

By Lemma 2.1 (with $\sigma=1$ ) we obtain a Euclidean quotient of $F$ of dimension $2^{-11} k^{\prime} \geq 2^{-12} k$ and so we complete the proof by passing to the dual.

Let us note that the one-sided boundness assumption alone still yields the existence of some Euclidean subspaces, but this time on a proportional level only.

Lemma 5.3 Let $0<\alpha<\left(1+2^{-12}\right)^{-1}$ and let $M \geq 1$. Let $G$ be an $n$ dimensional Banach space such that every an-dimensional subspace $E$ of $G$ has the basis constant $\mathrm{bc}(E) \leq M$. Then for every $\left(1+2^{-12}\right) \alpha<\lambda \leq 1$, every $\lambda$-dimensional subspace $G_{0}$ of $G$ contains a subspace $H$ with $\operatorname{dim} H=$ $(\lambda-\alpha) n \geq 2^{-13} \lambda n$ such that $\mathrm{d}_{H} \leq C(\alpha, \lambda) M^{2}$.

Proof A similar argument as in the proposition above, in which the use of (3.6) is replaced by (3.5), shows that every subspace $G_{1}$ of $G_{0}$ with $\operatorname{dim} G_{1}=$ $\xi n=\left(1+2^{-12}\right) \alpha n$ contains a $C M^{2}$-Euclidean subspace of dimension $2^{-11} \alpha n$, where $C$ is a universal constant. The proof is then concluded by applying Lemma 2.4, with $\delta=\left(1-2^{-12}\right) \alpha$ and $\eta=2^{-12} \alpha$, to any $\lambda n$-dimensional subspace $G_{0}$ of $G$.

An a priori argument which we are going to use is based on a finitedimensional version of one of properties characterizing weak type 2 spaces. A known argument ( $c f .[\mathrm{P} .3]$, Chapter 11), localized to a fixed proportionaldimensional level, gives the following lemma. Recall that for a Banach space $G, K(G)$ stands for the $K$-convexity constant of $G$.

Lemma 5.4 Let $0<\delta<\beta<1, D \geq 1$, and let $G$ be an $n$-dimensional Banach space. Assume that every $\beta n$-dimensional subspace $F$ of $G^{*}$ contains a subspace $F_{1}$ with $\operatorname{dim} F_{1}=\delta n$ and $\mathrm{d}_{F_{1}} \leq D$. Then for every $\beta n$-dimensional subspace $E$ of $G$ there exist a subspace $H$ with $\operatorname{dim} H=\delta n / 2$ and a projection $Q: G \rightarrow H$ such that $\|Q\| \leq C(\beta, \delta) K(G) D \mathrm{~d}_{E}$. 
Proof Let $E$ be a $\beta n$-dimensional subspace of $G$ and let $w: E \rightarrow l_{2}^{\beta n}$ be an isomorphism such that $\|w\|\left\|w^{-1}\right\|=\mathrm{d}_{E}$. We will show that there exist an orthogonal rank $(\delta / 2) n$ projection $P$ in $l_{2}^{\beta n}$ and an operator $\tilde{w}: G \rightarrow l_{2}^{\beta n}$, such that $\tilde{w}=P w$ and $\|\tilde{w}\| \leq C(\beta, \delta) D K(G)\|w\|$. Then $H=w^{-1} P\left(l_{2}^{\beta n}\right)$ and $Q=w^{-1} P \tilde{w}$ will satisfy the requirements of the lemma.

The argument requires the definition of the $l$-norm of an operator $u$ : $l_{2}^{k} \rightarrow Y$, for any Banach space $Y$, which is provided e.g. in [P.3], Chapter 3. Similarly as in the proof of Theorem 11.6 in $\mathbb{P} .3 \|$, consider the operator $w^{*}: l_{2}^{\beta n} \rightarrow G^{*} / E^{\perp}$. By Lemma 11.7 in $\left[\mathrm{P} .3\right.$, there exists $\tilde{v}: l_{2}^{\beta n} \rightarrow G^{*}$ such that $q \tilde{v}=w^{*}$, where $q: G^{*} \rightarrow G^{*} / E^{\perp}$ it the quotient map, and that $l(\tilde{v}) \leq 2 K(G) l\left(w^{*}\right) \leq 2(\beta n)^{1 / 2} K(G)\left\|w^{*}\right\|$. Note that $\tilde{v}$ is one-to-one. Choose a subspace $F_{1}$ of $\tilde{v}\left(l_{2}^{\beta n}\right)$ with $\operatorname{dim} F_{1}=\delta n$ and $\mathrm{d}_{F_{1}} \leq D$ and let $F_{2}=\tilde{v}^{-1}\left(F_{1}\right)$. By well-known properties of operators acting in Hilbert spaces (cf.e.g., $\mathrm{P} .3$ Proposition 3.13), there is a $(\delta / 2) n$-dimensional subspace $F_{3} \subset F_{2}$ and an orthogonal projection $P$ onto $F_{3}$ such that

$$
\|\tilde{v} P\| \leq(\delta n / 2)^{-1 / 2} D l(\tilde{v}) \leq 2^{3 / 2}(\beta / \delta)^{-1 / 2} K(G) D\|w\| .
$$

Therefore the required operator is $\tilde{w}=P \tilde{v}^{*}$.

We are finally ready for the proof of the theorem.

Proof of Theorem 5.1 Fix $0<\alpha<1 / 4$ and set $\alpha^{\prime}=\left(1+2^{-12}\right) \alpha$. By Lemma 5.3 with $\lambda=1$, pick a subspace $H$ of $G$ with $\operatorname{dim} H \geq(1-\alpha) n$ and $\mathrm{d}_{H} \leq C(\alpha) M^{2}$. Since all $\alpha$-dimensional quotients $F$ of $G$ satisfy $\operatorname{bc}(F) \leq$ $M$, applying the same lemma for $G^{*}$ and $\lambda=(1-\alpha)>\alpha^{\prime}$, it follows that $G^{*}$ satisfies the assumptions of Lemma 5.4 for $\beta=1-\alpha, \delta=1-2 \alpha$ and $D=C(\alpha) M^{2}$. Therefore there exist a subspace $H_{0}$ of $H$ and a projection $Q$ from $G$ onto $H_{0}$ such that $\operatorname{dim} H_{0}=(1 / 2-\alpha) n$ and $\|Q\| \leq C^{\prime}(\alpha) K(G) M^{4}$. Notice that $\operatorname{dim} H_{0} \geq \alpha n$.

Set $G_{0}=\operatorname{ker} Q$ and apply Proposition 5.2 to both $G$ and $G^{*}$ to conclude that the their weak cotype 2 constants satisfy

$$
\max \left(w C_{2}(G), w C_{2}\left(G^{*}\right)\right) \leq C^{\prime \prime}(\alpha)\|Q\|\left(\mathrm{d}_{H_{0}} M^{2}\right)^{4} \leq C^{\prime \prime}(\alpha) K(G) M^{20} .
$$

In particular, by (2.2), we also have

$$
w T_{2}(G) \leq K(G) w C_{2}\left(G^{*}\right) \leq C^{\prime \prime}(\alpha) K(G)^{2} M^{20}
$$


Now we use the result of Pisier stated in (2.3), for e.g., $\theta=1 / 6$, to get $K(G) \leq C^{\prime \prime \prime}(\alpha) K(G)^{1 / 2} M^{40 / 6}$. Thus

$$
K(G) \leq C_{0}(\alpha) M^{40 / 3}
$$

The proof is then concluded by combining this inequality with (5.1) and (5.2).

Remark Applying Lemma 5.3 with $\sigma$ arbitrarily close to 1 and Corollary 3.3 with $\varepsilon$ aufficiently small, one can replace the constant $1 / 4$ in Theorem 5.1 by any number smaller than 1 . Similar rearrangements yield more civilized powers of $M$.

\section{Random quotients}

Fix a probability space $(\Omega, \mathbf{P})$ and let $g_{1}, \ldots, g_{\varepsilon n}$ be independent standard Gaussian vectors in $\mathbb{R}^{n}$ with the density $(n / 2 \pi)^{n / 2} \mathrm{e}^{-n\|x\|_{2}^{2} / 2}$, with respect to the standard Lebesgue measure in $\mathbb{R}^{n}$.

For $\omega \in \Omega$, define a Gaussian projection $Q_{\omega}: \mathbb{R}^{N} \rightarrow \mathbb{R}^{n}$ by

$$
Q_{\omega}\left(e_{i}\right)= \begin{cases}e_{i} & \text { for } i=1,2, \ldots, n \\ g_{i-n}(\omega) & \text { for } i=n+1, n+2, \ldots, N .\end{cases}
$$

In the theorem below, we denote by $G_{\gamma n, n}$ the set of all $\gamma n$-dimensional subspaces of $\mathbb{R}^{n}$. For $H \in G_{\gamma n, n}$ we denote by $Q_{H}$ the orthogonal projection with $\operatorname{ker} Q_{H}=H$.

Theorem 6.1 For an arbitrary $0<\delta<1,0<\eta<3 / 8$ and $0<\varepsilon<2^{-5} \eta$, set $\gamma=2^{-5} \delta \varepsilon \eta$, and for $n \in \mathbb{N}$ set $N=(1+\varepsilon) n$. Let $E=\left(\mathbb{R}^{N},\|\cdot\|\right)$ be an $N$-dimensional Banach space such that $B_{E} \subset B_{2}^{N}$. Let $\rho \geq 1$ and $0<a \leq 1$ satisfy

$$
\operatorname{vr}(E) \leq \rho \quad a B_{2}^{N} \subset B_{E} .
$$

There exists $0<c=c(\varepsilon, \eta, \rho)<1$ such that if $\tilde{\Omega}$ denotes the set

$$
\begin{aligned}
\tilde{\Omega}=\{\omega \in \Omega \quad \mid & \left\|Q_{H} T: Q_{\omega}\left(l_{1}^{N}\right) \rightarrow Q_{H} Q_{\omega}(E)\right\| \geq c V_{\eta n / 4}(E)^{-1} a^{\delta}, \\
& \text { for every } \left.T \in \operatorname{Mix}_{n}(\eta n, 1) \text { and every } H \in G_{\gamma n, n}\right\},
\end{aligned}
$$

then $\mathbf{P}(\tilde{\Omega}) \geq 1-c_{1}^{n^{2}}$, where $0<c_{1}<1$ is an absolute constant. 
To deduce Theorem 3.1 pick $\omega \in \tilde{\Omega}$ and set $F=Q_{\omega}(E)$. Now it is enough to observe that since $\kappa B_{1}^{N} \subset B_{E}$ then

$$
\left\|Q_{H} T: Q_{\omega}(E) \rightarrow Q_{H} Q_{\omega}(E)\right\| \geq \kappa\left\|Q_{H} T: Q_{\omega}\left(l_{1}^{N}\right) \rightarrow Q_{H} Q_{\omega}(E)\right\| .
$$

For $E=l_{1}^{N}$, Theorem 6.1 was proved recently in M-T.2 Theorem 4. In the general case the argument follows the steps from M-T.2 blended with a technique which enables to pass from quotients of $l_{1}^{N}$ to quotients of arbitrary Banach spaces, as presented in [M-T.3], Section 5. Therefore we shall only briefly discuss the main points, referring the reader to M-T.2 and M-T.3 for the details.

Passing to the description of the proof of Theorem [6.1, we require additional notation. For every $\omega \in \Omega$ let $H_{\omega}=\operatorname{span}\left[g_{1}(\omega), g_{2}(\omega), \ldots, g_{\varepsilon n}(\omega)\right]$. If $H \in G_{\gamma n, n}$, let $Q_{\omega, H}$ be the orthogonal projection in $\mathbb{R}^{n}$ with $\operatorname{ker} Q_{\omega, H}=$ $H+H_{\omega}$.

Let

$$
\Omega_{0}=\left\{\omega \in \Omega \mid 1 / 2 \leq\left\|g_{i}(\omega)\right\|_{2} \leq 2 \text { for all } i=1, \ldots, \varepsilon n\right\} .
$$

Fix $T \in \operatorname{Mix}_{n}(2 \eta n / 3,1)$. By the definition of the mixing class, there is $G \subset \mathbb{R}^{n}, \operatorname{dim} G=2 \eta n / 3$ such that $\left\|P_{G^{\perp}} T x\right\|_{2} \geq\|x\|_{2}$ for every $x \in G$. The well-known argument on half-dimensional circular sections of an ellipsoid yields that there exists $G_{0} \subset G$ with $\operatorname{dim} G_{0}=\eta n / 3$ and $\lambda \geq 1$ such that $\left\|P_{G^{\perp}} T x\right\|_{2}=\lambda\|x\|_{2}$ for every $x \in G_{0}$. For every $\omega \in \Omega$ and $H \in G_{\gamma n, n}$ fix an orthogonal projection $Q_{\omega, H, G_{0}}$ in $\mathbb{R}^{n}$ with

$$
\operatorname{ker} Q_{\omega, H, G_{0}} \supset H_{\omega}+H+G+P_{G^{\perp}} T P_{G_{0}^{\perp}}\left(H_{\omega}\right) \text {, }
$$

and $\operatorname{rank} Q_{\omega, H, G_{0}}=\eta n / 4$.

Set

$$
\begin{aligned}
\Omega_{T, H}=\left\{\omega \in \Omega_{0} \quad\right. & Q_{\omega, H, G_{0}} T P_{G_{0}} g_{j} \in 4 \lambda \alpha \sqrt{\eta} a^{\delta} V_{\eta n / 4}^{-1} Q_{\omega, H, G_{0}} Q_{\omega}\left(B_{E}\right) \\
& \text { for } j=1,2, \ldots, \varepsilon n\} .
\end{aligned}
$$

Lemma 6.2 Let $H \in G_{\gamma n, n}$. Then

$$
\mathbf{P}\left(\Omega_{T, H}\right) \leq\left(C_{0} \alpha \sqrt{\eta} a^{\delta}\right)^{\varepsilon \eta n^{2} / 4},
$$

where $C_{0} \geq 1$ is an absolute constant. 
Proof Set $\widetilde{Q}_{\omega}=Q_{\omega, H, G_{0}}$ for $\omega \in \Omega$. For $j=1,2, \ldots, \varepsilon n$ define $g_{j}^{\prime}=P_{G_{0}} g_{j}$ and $g_{j}^{\prime \prime}=P_{G_{0}^{\perp}} g_{j}$. Similarly as in M-T.2 , Lemma 7, $\widetilde{Q}_{\omega}$ is independent of the $g_{j}^{\prime}$ 's. For every fixed $j=1,2, \ldots, \varepsilon n$ we have

$$
\begin{aligned}
& \left\{\omega \in \Omega \mid \widetilde{Q}_{\omega} T P_{G_{0}} g_{j} \in 4 \lambda \alpha \sqrt{\eta} a^{\delta} V_{\eta n / 4}^{-1} \widetilde{Q}_{\omega} Q_{\omega}\left(B_{E}\right)\right\} \\
& \quad=\left\{\omega \in \Omega \mid \widetilde{Q}_{\omega} T g_{j}^{\prime} \in 4 \lambda \alpha \sqrt{\eta} a^{\delta} V_{\eta n / 4}^{-1} \widetilde{Q}_{\omega} Q_{\omega}\left(B_{E}\right)\right\} .
\end{aligned}
$$

Since $G \subset \operatorname{ker} Q_{\omega}$, the definition of $\lambda$ implies that $\lambda^{-1} \widetilde{Q}_{\omega} T$ is a contraction in the Euclidean norm on $\mathbb{R}^{n}$. Moreover, it has $k s$-numbers equal to 1 , with $k \geq \eta n / 3-\gamma n-2 \varepsilon n \geq \eta n / 4$. Hence, using Claim 6.2 in [Sz.1] (with $n / 3$ replaced by $\eta n / 4$ ), and the fact that $\sqrt{3 / \eta} g_{j}^{\prime}$ is a standard Gaussian variable in $G_{0}$, (note that $3 / \eta=n / \operatorname{dim} G_{0}$ ), cf.e.g.,[Sz.1] (3.3), we have, by the definition of $V_{\eta n / 4}$,

$$
\begin{aligned}
& \mathbf{P}\left(\left\{\omega \in \Omega \mid \widetilde{Q}_{\omega} T g_{j}^{\prime} \in 4 \lambda \alpha \sqrt{\eta} a^{\delta} V_{\eta n / 4}^{-1} \widetilde{Q}_{\omega} Q_{\omega}\left(B_{E}\right)\right\}\right) \\
& \quad \leq \mathbf{P}\left(\left\{\omega \in \Omega \mid\left(\lambda^{-1} \widetilde{Q}_{\omega} T\right)\left(\sqrt{3 / \eta} g_{j}^{\prime}\right) \in 4 \sqrt{3} \alpha a^{\delta} V_{\eta n / 4}^{-1} \widetilde{Q}_{\omega} Q_{\omega}\left(B_{E}\right)\right\}\right) \\
& \quad \leq\left(c^{\prime} 4 \sqrt{3} \alpha a^{\delta}\right)^{\eta n / 4} .
\end{aligned}
$$

where $c^{\prime}$ is an absolute constant. Hence

$$
\mathbf{P}\left(\Omega_{T, H}\right) \leq\left(c^{\prime} \alpha a^{\delta}\right)^{\varepsilon \eta n^{2} / 4},
$$

which concludes the proof of the lemma.

The next lemma is a restatement of Lemma 7.3 in [Sz.1].

Lemma 6.3 For every $0<\sigma<1$, the set

$$
\mathcal{P}_{k, n}=\left\{P: \mathbb{R}^{n} \rightarrow \mathbb{R}^{n} \mid P \text { an orthogonal projection with } \operatorname{rank} P=n-k\right\}
$$

admits a $\sigma$-net $\mathcal{M}$ in the operator norm in $l_{2}^{n}$ with the cardinality $|\mathcal{M}| \leq$ $C^{n^{2}} \sigma^{-n k}$, where $C>1$ is an absolute constant.

Using Lemma 6.2 and Lemma 6.3 with $\sigma=\alpha a^{1+\delta} / 4$ and $k=\gamma n$, the same argument as in the proof of Proposition 5 in M-T.2 yields. 
Proposition 6.4 Let $0<\alpha, \delta<1$ and $0<\eta<3 / 8$. For an operator $T \in \operatorname{Mix}_{n}(2 \eta n / 3,1)$ set

$$
\begin{aligned}
\Omega_{T}=\left\{\omega \in \Omega_{0} \quad \mid\right. & \left\|Q_{\omega, H} T: Q_{\omega}\left(l_{1}^{N}\right) \rightarrow Q_{\omega, H} Q_{\omega}(E)\right\| \leq 2 \alpha \sqrt{\eta} a^{\delta} V_{\eta n / 4}^{-1} \\
& \text { for some } \left.H \in G_{\gamma n, n}\right\} .
\end{aligned}
$$

Then for every $T \in \operatorname{Mix}_{n}(2 \eta n / 3,1)$ one has

$$
\mathbf{P}\left(\Omega_{T}\right) \leq C^{n^{2}}\left(4 / a^{2} \alpha\right)^{\gamma n^{2}}\left(C_{0} \alpha \sqrt{\eta} a^{\delta}\right)^{\varepsilon \eta n^{2} / 4}
$$

where $C \geq 1$ and $C_{0} \leq 1$ are absolute constants.

The rest of the proof of Theorem 6.1 is essentially the same as of Theorem 4 in M-T.2]. One has to replace Proposition 6 there by Lemma 5.3 from

M-T.3, with $A=2 \alpha \sqrt{\eta} a^{\delta} V_{\eta n / 4}^{-1}$ and choosing $\alpha>0$ sufficiently small as to ensure that $(4 c(\rho))^{1+\varepsilon^{2}} C C_{0}^{\varepsilon \eta / 4}(\alpha)^{\varepsilon \eta / 8} \leq 1 / 2$.

\section{References}

[B] Bourgain, J., On finite-dimensional homogeneous Banach spaces. GAFA Israel Seminar 1986-87, Lecture Notes in Math., Vol. 1317, Springer, 232-239.

[B-S] Bourgain, J. \& Szarek, S. J., The Banach-Mazur distance to the cube and the Dvoretzky-Rogers factorization. Israel J. of Math., 62 (1988), 169-180.

[Ma.1] Mankiewicz, P., Finite dimensional Banach spaces with symmetry constant of order $\sqrt{n}$. Studia Math., 79, (1988), 193-200.

[Ma.2] Mankiewicz, P., Subspace mixing properties of operators in $\mathbb{R}^{n}$ with applications to Gluskin spaces. Studia Math., 88 (1988), 5167.

[Ma.3] Mankiewicz, P., Factoring the identity operator on a subspace of $l_{\infty}^{n}$. Studia Math., 95 (1989), 133-139.

[M-T.1] Mankiewicz, P. \& Tomczak-Jaegermann, N., A solution of the finite-dimensional homogeneous Banach spaces problem. Israel J. Math. 75 (1991), 129-159. 
[M-T.2] Mankiewicz, P. \& Tomczak-Jaegermann, N., Embedding subspaces of $l_{\infty}^{n}$ into spaces with Schauder basis. Proc. of the AMS 117 (1993), 459-465.

[M-T.3] Mankiewicz, P. \& Tomczak-Jaegermann, N., Schauder bases in subspaces of quotients of $l_{2}(X)$. To appear in Amer. J. of Math.

[Mi] Milman, V. D., Inégalité de Brunn-Minkowski inverse et applications le théorie local des espaces normés. C. R. Acad. Sci. Paris, 302 Sér. 1 (986), 25-28.

[M-P] Milman, V. D. \& Pisier, G., Banach spaces with weak cotype 2 property. Israel J. Math. 54 (1986), 139-158.

[Pe.1] Pełczyński, A., Notes in Banach spaces. (H. E. Lacey ed.), Univ. of Texas Press, Austin 1980.

[P.1] Pisier, G., Holomorphic semi-groups and the geometry of Banach spaces. Annals of Math. 115 (1982), 375-392.

[P.2] Pisier, G., On the duality between type and cotype, Martingale Theory in Harmonic Analysis and Banach spaces, Proceedings, Cleveland 1981 (ed. J.A. Chao and W. A. Woyczynski), Springer Lecture Notes No. 939, 131-144.

[P.3] Pisier, G., Volumes of Convex Bodies and Banach Spaces Geometry. Cambridge Univ. Press, 1989.

[Sz.1] Szarek, S. J., The finite dimensional basis problem with an appendix on nets of Grassmann manifolds. Acta Math., 151 (1983), 153-179.

[Sz.2] Szarek, S. J., On the existence and uniqueness of complex structure and spaces with "few" operators. Trans. AMS 293 (1986), 339-353.

[T] Tomczak-Jaegermann, N., Banach-Mazur Distances and Finite Dimensional Operator Ideals. Pitman Monographs and Surveys in Pure and Applied Mathematics, Longman Scientific \& Technical, Harlow and John Wiley, New York, 1989. 
Institute of Mathematics,

Polish Academy of Sciences

Śniadeckich 8,

00-950 Warsaw, Poland,

piotr@impan.impan.gov.pl

Department of Mathematics,

University of Alberta,

Edmonton, Alberta, Canada T6G 2G1,

ntomczak@vega.math. ualberta.ca 